\title{
Russian/Former Soviet Union Experience in Professional Small Telescope Usage
}

\author{
N.G. Bochkarev \\ Euro-Asian Astr.Soc., Sternberg Astr.Inst.(SAI), Universitetskij \\ Prospect 13, Moscow119899, Russia; boch@sai.msu.ru
}

FSU astronomers traditionally use small telescopes $(\varnothing \leq 1.5 \mathrm{~m}$, hereafter STs) for both science and education. Russian/FSU experience here is among the largest world-wide. There are only 2 large and moderate-sized facilities in whole Russia: the $6 \mathrm{~m}$ telescope of SAO RAS and Russian-Ukrainian $2 \mathrm{~m}$ one on the $3100 \mathrm{~m}$ high peak Terskol in Central Caucasus.

Equipped with good light receivers and handled by skilled observers, STs can produce first class scientific data. Important results are typically yielded by long-time sequences of observations and/or new observational "know how": good instrument/receiver design, appropriate selection of objects and moments, etc. Examples of what has been done with STs in FSU, within my memory, (in the last $\simeq 1 / 3$ century) are listed below, without a list of references, because of lack of space. The author plans to publish a larger article on this subject in Astr.\&Aph.Trans.

\section{Solar System Objects}

Meteors. S.Mukhamednazarov obtained a unique sequence of $>200$ spectra of $2-6^{m}$ meteors with a small $(\approx 5 \mathrm{~cm})$ aperture wide-field camera $\left(20 \times 20^{\circ}\right)$ supplied with a slitless spectrograph and a TV light receiver. The observations, carried on at Turkmenistan since 1972, trebled the number of spectra obtained during the previous 75 yrs, moved the observational limit to $3-4^{m}$ dimmer events, and contributed considerably to meteor chemical composition research.

Comets. Monitoring of comet tails (their size, shape, brightness, structure: non-stationary processes, e.g. tail-breaks) with STs to search for interplanetary magnetic field and solar activity influence is yet an important task for amateurs and professionals. High-quality images come from places with good seeing: Mt. Maidanak Obs. (Uzbekistan), Mt. Sanglok Obs. (Tajikistan), Mt.DushakEregdag Obs. (Turkmenistan) and others.

Spectroscopy is easily done with STs for bright comets. K.Churyumov (AO of Kiev State Univ.(SU), Ukraine) \& colleagues have obtained good spectra (resolution of $\approx 50000$ ) with $1 \mathrm{~m} \mathrm{ST}+\mathrm{CCD}$-camera. This is a valuable input into the gas chemical and isotopic composition and outflow parameter study.

Positional astronomy. Missions to planet satellites and asteroids required a considerable improvement of ephemerides accuracy. Examples of ST usage for this purpose are:

1. S.Novikov \& A.Shokin (SAI) performed, in 1987, >1000 positional measurements of Phobos and Daimos from Mt. Maidanak (a place with very good 
seeing - see A.Hojaev in this volume), with a $1 \mathrm{~m}$ ST equipped with a screen for Mars light eclipsing, and a $0.23 \mathrm{~m}$ wide-field astrograph designed in $1847(!)$ and moved to $\mathrm{Mt}$. Maidanak in the 1970s. Thanks to this research the resulting pointing deviation for the ship "Phobos" was only $2 \mathrm{~km}$.

2. Ephemerides for Jupiter and Saturn satellites have been improved through photometric observation campaigns of their rare mutual occultations and eclipses. The 1990s campaign supervised by N. Emel'yanov (SAI) involved about 10 teams of FSU observers that used 0.3-0.8 $\mathrm{m}$ STs. Even visual observations by an SAI student, A.Berezhnoj, with a monocular $(\oslash=6 \mathrm{~cm} ; \mathrm{f}=30 \mathrm{~cm})$ were a great input similar to those by a ST+CCD-camera.

3. Stellar occultations by asteroids.

Asteroids. In 1965-1995 N.\&L.Chernykh, at Crimean Aph.Obs. (CrAO, Ukraine), using a wide-field $40 \mathrm{~cm}$ astrograph made the world-wide largest input in asteroids discovery: $\approx 50 \%$ of the asteroid discoveries in the world and a great impact on the asteroid hazard problem. They named about 800 asteroids.

V.Prokof'eva's group (CrAO) using a TV 2D-receiver (I-Isocon) with a 50 $\mathrm{cm} \mathrm{ST}$ and $5 \mathrm{~min}$. exposure measures objects of $19-20^{m}$ with a $5 \%$ accuracy. Such observations yield important data: besides rotation and shape determination, binary asteroids were discovered, and precession of one of them detected.

Asteroid stellar occultations are another important field of ST application: knowledge of asteroid orbits, shapes, and the accuracy of some physical parameters may be increased.

B.Artamonov (SAI) with V.Dudinov (AO of Kharkov SU, AO KhSU, Ukraine) et al., obtained at Mt. Maidanak, using speckle-interferometry and a $1 \mathrm{~m} \mathrm{ST}$, a direct image of Vesta (angular size $\approx 0.5 "$ ) with a resolution of $0.13 "$.

The mineral composition of some asteroids was estimated by S.Omarov et al. from spectra obtained with a $0.7 \mathrm{~m} \mathrm{ST}$ of Shemakha Aph. Obs. (Azerbaijan) in $1980 \mathrm{~s}$.

Planets. The great and rare opportunity to observe the Shoemaker-Levy comet impact on Jupiter was successfully used by many observers with STs. On June 20, 1994, K.Churyumov with the $0.5 \mathrm{~m}$ ST of Lesniki Obs. near Kiev and V.Prokof'eva $(\mathrm{CrAO})$ with 50 and $20 \mathrm{~cm} \mathrm{ST}$ measured an $\approx 1 \mathrm{~s}$ long flash $\left(\approx 0.1^{\mathrm{m}}\right)$ produced by the fragment Q2 crashing into an invisible part of the planet's atmosphere and reflected by Io. V.Prokof'eva obtained, on Jul. 16 - Aug. 30, 1994, $>500$ spectral and UBV measurements of the fireballs produced by the falling comet fragments. NaI doublet emission allowed investigations of the interaction of comet matter with the Jovian magnetosphere plasma. B.Artamonov et al., at Mt. Maidanak, obtained high angular resolution images of the fireballs.

V.Prokof'eva performed, with a $0.5 \mathrm{~m} \mathrm{ST}$ and a TV-receiver, spectroscopic observations of $\mathrm{NaI}$ in the Jovian atmosphere. She and V.Lyuty \& V.Tarashchuk, with a $60 \mathrm{~cm}$ ST, studied in detail Pluto's UBV variation with its rotational period.

Moon. SAI, Abastumani Aph.Obs. (AAO, Georgia) \& AO KhSU (Ukraine) have great experience in the Moon surface structure and mineralogy analysis by polarimetric mapping with STs. 


\section{2. "Classic" Fields of Stellar Astronomy}

Optical sky patrol has been carried on for decades at many observatories with 5-50 cm STs. The largest photoplate archives belong to SAI, AO of Odessa Univ. (OAO, Ukraine), and the Inst. for Astr. of Tajik Acad.Sci (IA TAS). The plates are intensely used for research in the history of brightness of variable objects: CV-stars, X-ray binaries, AGN, QSO, gamma-ray bursts (GRB), etc. (see below), the largest experience here being that of SAI \& OAO. Presently, plate collecting and wide-field sky patrols are canceled, but D.Tsvetkov (SAI) continues SN patrol using wide-field cameras and plates or CCD-cameras.

Proper motion of stars was measured with photoplates exposed at distant epochs at SAI, Pulkovo, OAO, Main AO of NAS Ukraine (MAO, Kiev) etc.

Photometry of variable stars is a rich field for both professionals and amateur work with STs. With the amateurs, AAVSO experience is the largest.

Many scientists-from SAI MSU (pupils of P.P.Parenago, B.V.Kukarkin, D.Ya.Martynov: A.Cherepashchuk, V.Goranskii, R.i Khaliullin, V. Lyuty, N. Samus, S.i Shugarov et al.), OAO (pupils of V.P. Tsessevich: I. Andronov, D. Mkrtichian, others), and Kazan SU (pupils of A.Dubyago: M. Lavrov, E. Zhukov, others) got numerous brilliant results partially listed below.

Cepheids. 55000 photometric observations of 369 Cepheids ( $>50 \%$ of the world data archive) have been taken by L.Berdnikov (Saratov SU/SAI) at Mt. Maidanak, Las Campanas, Cerro Tololo \& South Africa, with the 0.48-1 m ST. Prior to this work, about 150 Cepheides had never been observed photoelectrically; data on 100 Cepheids were poor. The results have had an important impact on the determination of the Galaxy distance scale and structure, and in the investigation of Cepheid period variations, i.e. stellar evolution.

To specify globular clusters distance scale, V.Goransky et al. observed the clusters RR Lyr type stars $\left(B=19-20^{m}\right)$ light curves with a $1 \mathrm{~m} \mathrm{ST}$ at Tian-Shan Mt. Obs. The $>30000$ observations of RR Lyr type stars have been collected in OAO.

Orbital period variations. Long-time sequences of data on variable stars by M.A.Svechnikov and his pupils (Ural SU) have resulted in the production of a catalogue submitted to Strasbourg Data Center. They found impressive examples of fast changes of orbital periods, $P_{o}$, in binaries which indicate episodes of intensive mass eruptions.

Relativistic effects in classical binaries (periastron shifting) were detected and investigated by late D.Martynov \& R.Khaliullin et al. with ST at SAI Cr.Lab.

Novae and nova-like stars. The brightest Nova, N Cygni 1975 (V1500 Cyg) discovered visually by S.Shugarov (SAI) was studied at CrAO by E.Pavlenko et al. with a $50 \mathrm{~cm}$ ST. Its post-nova stable state $\left(\mathrm{V}=17.2^{\mathrm{m}}\right)$ light curve was analyzed in detail; after outburst the orbital period increased on a time-scale of 230 yrs.

Using the 60-cm SAI Cr.Lab. ST, I.Voloshina found the low state photometric periods for the dwarf novae V795 Her, AH Her, and AB Dra. Observations of the dwarf nova, SS Cyg, including outbursts fast photometry have been carried on for 10 years. Quasi-periodic oscillations have been registered and independently studied by Yu.Gnedin et al. (Pulkovo AO, St.Peterburg) using AAO ST observations. 
Symbiotic stars are studied in CrAO starting with the cycle of papers by A.Boyarchuk \& T.Belyakina in the 1960s which showed their physical nature. We should also mention the detailed search of CH Cyg by L.Luud (IAPA, Estonia), IR photometric observations of a sample of the objects and monitoring of V1016 Cyg symbiotic nova and other peculiar objects carried on by O.Taranova's group (SAI) with InSb receiver at $1.25 \mathrm{~m} \mathrm{ST}$ in SAI Cr.Lab. as well as spectral and photometric observations by V.Arkhipova, V.Esipov et al. (SAI) using the same ST.

Young stars. More than 70000 UBVR observations $(\approx 230$ stars $)$ of $T$ Tau type and Herbig Ae/Be young stars and FUORs were taken at Mt. Maidanak with the $48 \mathrm{~cm}$ and $60 \mathrm{~cm}$ STs over 20 yrs (long-term program) by the group of late V.Shevchenko (K.Grankin, O.Ezhkova, S.Mel'nikov). Star spots were studied, new subclasses of proto-Algols and quasi-Algols discovered, information on proto-planetary matter around stars were gathered.

Fine results for UV Ceti stars were obtained at BAO (Armenia), CrAO (R.Gershberg et al.), and Mt. Maidanak (V.Shevchenko et al., Astr.Inst., Uzbekistan).

Lunar occultations fast photometry is a rich field for STs even for $\oslash=0.3-0.4$ $\mathrm{m}$. The observations can yield star diameters (or their upper limits), and help to discover/study close binaries (distance between components $\approx 0.005-1$ "). In Russia the best results have been obtained by E.Trunkovsky (SAI).

Double stars. A.Tokovinin has determined, through observations with STs of 0.6-1 m and an original hand-made photoelectric phase-grating interferometer positional angles and separations of several hundreds of double stars. The rms measurement error is $\approx 0.007 "$ (at $1 \mathrm{~m} \mathrm{ST}$ ). The results form a considerable input into statistics of individual double system orbits, masses and mass ratios, i.e. for the determination of fundamental properties and leading to an understanding of the formation process of stars.

Spectrophotometric catalogues and robotic telescopes. Bright star absolute spectrophotometry for catalogue creation has carefully been carried out at SAI (I.Glushneva, V.Kornilov et al.), AFIF (A.Kharitonov et al.), and OAO (N.Komarov et al.) etc.

Twin $1 \mathrm{~m}$ robotic telescopes were installed in the $1980 \mathrm{~s}$ by SAI at TianShan Mt. Obs. $(2700 \mathrm{~m})$ for such catalogue production and for studying optical counterparts of radio/X-ray sources. These STs do not presently function.

Radial velocities. Exciting results were achieved with $0.5-1 \mathrm{~m}$ STs in stellar radial velocity study with COROVEL-spectrometer designed by A.Tokovinin even with as bad seeing conditions as in Moscow. See the paper by N.Samus in this volume.

High precision star photometry, even with a relatively bad seeing/weather has been successfully realized at the $70 \mathrm{~cm} \mathrm{ST}$ of Ural SU Kourovskaya AO with a guided four-beam CCD photometer. The device yields a precision of $0.005^{m}$ or better. An accuracy of $0.0015^{m}$ was achieved in observations of the planetary transit across the disc of the star HD209458.

The device permits to separate a flux of close double star components, e.g. allowed to get, for the 1st time, parameters of the triple variable AM Leo.

One example of the study of very low amplitude variability $\left(\approx 0.008^{m}\right)$ is V.Goransky et al.'s (1999) study of V1674 Cyg used as a standard for obser- 
vations of Cyg X-1. High accuracy observations were achieved thanks to the highly stable atmosphere transparency at Tian-Shan and Maidanak Mt. Obs.

Stellar polarimetry. Famous results were achieved with STs in FSU in this area. Many researchers are/were involved: N. Shakhovskoy (who discovered intrinsic variable stellar polarization in 1962) \& Yu.Efimov in CrAO; Yu. Gnedin et al. (Pulkovo AO); A. Kurchakov et al. (AFIF, Kazakhstan); V. Dombrovskij's pupils in SPbSU (Russia); M. Vashakidze's pupils in AAO (Georgia), V. Oskanian's pupils in BAO (Armenia), IA TAS (Tajikistan) etc.

\section{Modern Stellar Astronomy}

Optical monitoring of X-ray binaries. Since X-ray binaries were discovered by UHURU at the beginning of the 1970s, their optical identification on archive photoplates and photometric history study were started at SAI. Since then V.Lyuty has performed optical monitoring of many such objects with a $60 \mathrm{~cm} \mathrm{ST}$. Even the preliminary results allowed to determine the nature of optical variability, which differs for different objects. The lower mass limit of the invisible X-ray component of the first black hole candidate, Cyg X-1, proved that the object could not be a neutron star. Further data gathering and analysis revealed many fine details in the structure and light curves and lead to an understanding of physical processes within the objects.

A six month long set of observations of a number of southern X-ray binaries by A.Cherepashchuk in Australia with a $60 \mathrm{~cm}$ ST proved that long-period variability, the so called precession effects in accretion disks $\left(P_{p r}=35^{d}\right.$ for Her $\mathrm{X}-1$ and $164^{d}$ for SS 433 ) is quite typical for X-ray binaries.

Detailed study of the light curves allowed to determine the fundamental parameters of the system: the componentent masses, the orbit size and inclination. A large contribution to the understanding of the nature of SS 433 was made by A.Cherepashchuk et al. First of all, the light curve was studied in detail using data from the Mt. Maidanak 0.6 and $1 \mathrm{~m} \mathrm{STs.} \mathrm{Dense} \mathrm{sequences}$ of multi-color observations over several $164^{d}$ cycles allowed to trace the orbital light curve variations with the cycle phase and set limits on the geometry of the system.

Deviations from average light curves (first of all, flashes) allow to study non-stationary accretion processes and gas behavior at the accretion disks outer rim. Presently the best studied case is Cyg X-1. Intensive high-accuracy UBV monitoring with the 0.6-1 m ST at Maidanak and Tian-Shan Mt. has permitted E.Karitskaya (INASAN) et al. to find and analyze optical flashes $\left(\leq 0.04^{m}\right)$ and, for the first time, compare them with X-ray data from RXTE/ASM and find time lag of X-ray events relative to the optical ones $\left(7-12^{d}\right)$ which is the time interval of the matter flow from the outer to the inner rim of the disk. At SAI Cr.Lab. $(0.6 \mathrm{~m} \mathrm{ST})$ the largest flash in the last 30 yrs was registered $\left(\approx 0.12^{m}\right)$ pointing to explosion-type gas outflow from the disk's outer rim (N.Bochkarev \& V.Lyuty, SAI, 1998).

Cooperative programs of Cyg X-1 observations in different spectral ranges set in action by V.Lyuty (UBVR data from $0.6 \mathrm{~m} \mathrm{ST}$ ) detected $P_{p r}$ in radio-, optic and X-ray ranges, which do not contradict to earlier optic data of J.Kemp 
(USA) with E.Karitskaya, V.Lyuty et al. and others, and permit to include Cyg $\mathrm{X}-1$ in the list of so-called mini-quasars.

In the $1980 \mathrm{~s}$ detailed precision $\left(0.002^{m}\right)$ photometry of Her X-1 carried out by N.Kilyachkov \& V.Shevchenko (AI, Uzbekistan) with the Mt. Maidanak $60 \mathrm{~cm}$ ST revealed, during the X-ray source occultation by the optical component, a series of its light curve peculiarities in the minimal brightness stage. N.Shakhovskoy \& Yu.Efimov with CrAO $1.25 \mathrm{~m}$ ST detected, during that same period, flashes of polarization. Peculiarities of the Her X-1 light curve near the minima are being studied with STs of SAI Cr.Lab. The data is still being discussed by theorists.

Stimulating UBV observations of X-ray binaries were carried out with the $1 \mathrm{~m} \mathrm{ST}$ at Sanglok Mt. Obs. IA TAS by R.Rakhimov in the 1980s.

Polarimetry. N.Shakhovskoy \& Yu.Efimov performed 5-color polarimetry of a number of X-ray binaries with CrAO $1.25 \mathrm{~m} \mathrm{ST}$ : SS 433, Cyg X-1, polars, etc. Magnetic fields of magnetized white dwarfs (WD) were measured and accretion on WD investigated.

GRB. Late E.Moskalenko (SAI) together with OAO, before the BeppoSAX launch in 1997, searched for optical counterparts of GRBs using the archive photoplates, mainly, of the OAO 7-cameras ST and, a larger number of artifacts were excluded, revealing non-trivial events that could be short ( $\leq 40 \mathrm{~min}$.) optical flashes.

Gravitational microlensing is now being widely discussed. Unfortunately, in Russia, the work on the topic is mainly theoretical.

Asteroseismology is a very prospective trend of ST usage. As high precision $24^{h}$-round monitoring is required, investigations need to be conducted with wide international cooperation. An example of this type of network initiated and put into action, in particular, by FSU astronomers is the Central Asian Network (CAN) organized by D.Mkrtichian (OAO) with co-investigators from Russia, Georgia, Kazakhstan, and Turkmenistan; another branch includes WestEuropean and US colleagues. In 1996, 29 Cyg observation global campaign 10 oscillation modes were detected.

For interstellar matter/nebulae see Bochkarev's paper in this volume.

\section{Extragalactic Objects}

Photometry. Since the late 1960s V.Lyuty (SAI) using a $60 \mathrm{~cm} \mathrm{ST}$, has been carrying on a long term program of UBV-photometry of many AGN. He investigated many outbursts (characteristic time 10-30 ${ }^{d}$ ) re-occurring about every $100^{d}$ and discovered quasi-periodic brightness variations (characteristic time 1-3 yrs). By comparison with archive data from SAI plate collection, AGN variation cycles were traced over a time interval of up to 80 years. He studied minor brightness variations over one night. This had an important impact on research of the nature of activity, first of all, stability and variability of accretion disc parameters.

Polarimetry in UBVRI bands of BL Lac type objects was performed by N.Shakhovskoy (CrAO) \& Yu.Efimov with a $1.25 \mathrm{~m} \mathrm{ST}$. They showed that the linear polarization of some objects may reach $40 \%$ in some cases and is highly variable. 
Spectra. A unique low $\mathrm{S} / \mathrm{N}$ ratio spectra archive was collected by E.Denissiuk (AFIF, Kazakhstan) with a $70 \mathrm{~cm}$ ST and an image tube. In 1968-2000, thousands of spectra of 1515 galaxies were taken, emission lines in 48 galaxies and 42 new SyG were discovered. Vast amounts of material on spectral variability of 50 galaxies ( $>3000$ spectra) has been collected over 30 years. This archive is unique in the world.

Moderate to high S/N (20-100) AGN spectral monitoring is being carried out by N.Bochkarev (SAI), A.Shapovalova (SAO) \& others with a $1 \mathrm{~m}$ telescope of SAO RAS and a CCD-spectrograph (see N.Bochkarev \& A.Shapovalova, this volume). For the first time, an attempt to determine the lag of broad-line variations with respect to the continuum was made by A.Cherepashchuk and V.Lyuty in 1973 with the $0.6 \mathrm{~m}$ ST of SAI Cr.Lab.

High resolution images of cosmological gravitational lensing were obtained by B.P.Artamonov (SAI), V.Dudinov (AO KhSU) et al. at Mt. Maidanak Obs. The images with deconvolution allow to obtain Einstein Cross images with a resolution higher than 0.5 ", which is the world-wide best ground-based result, matching those by the HST. B.Artamonov \& V.Oknyansky (SAI) studied variations of its components brightness over $\approx 5$ yrs with the aim of finding the time lag of one component of the image with respect to the others. 


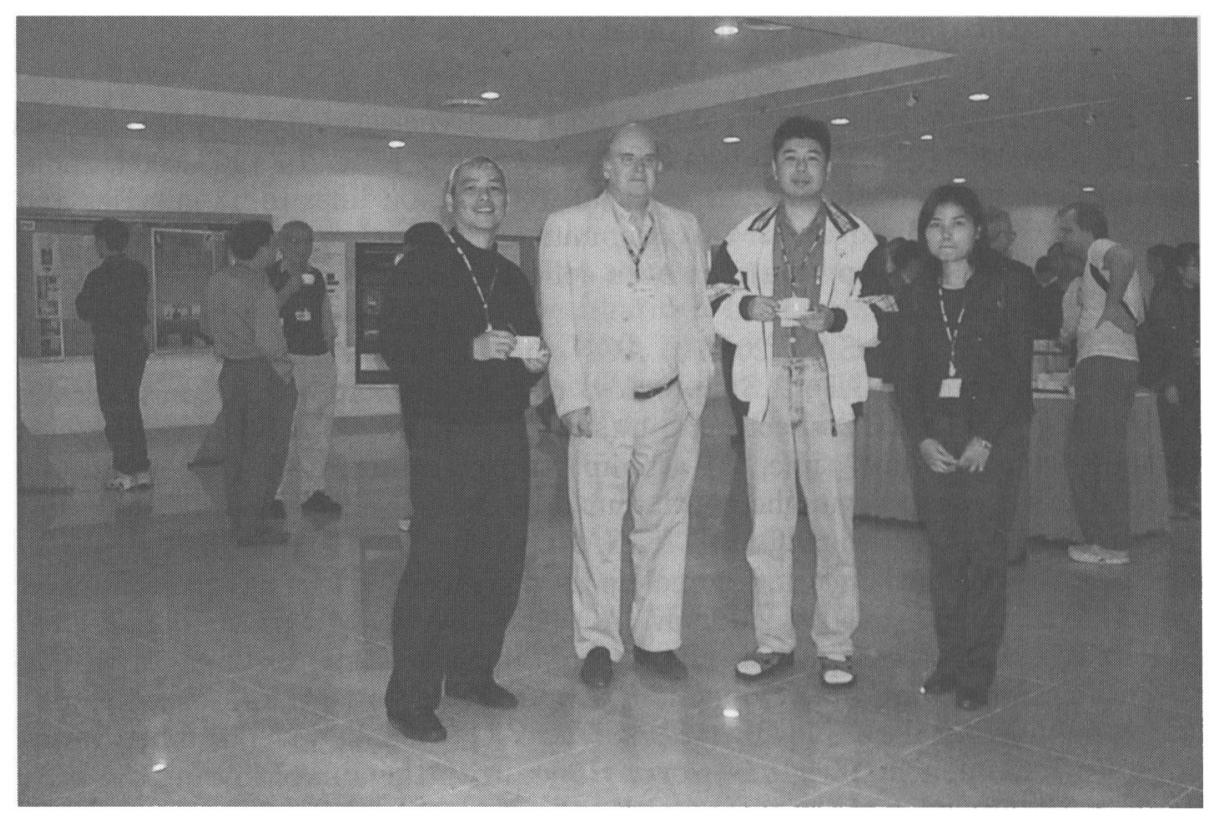

(Front from left) Fu, Warner, A.-L. Chen, J. H. Wang

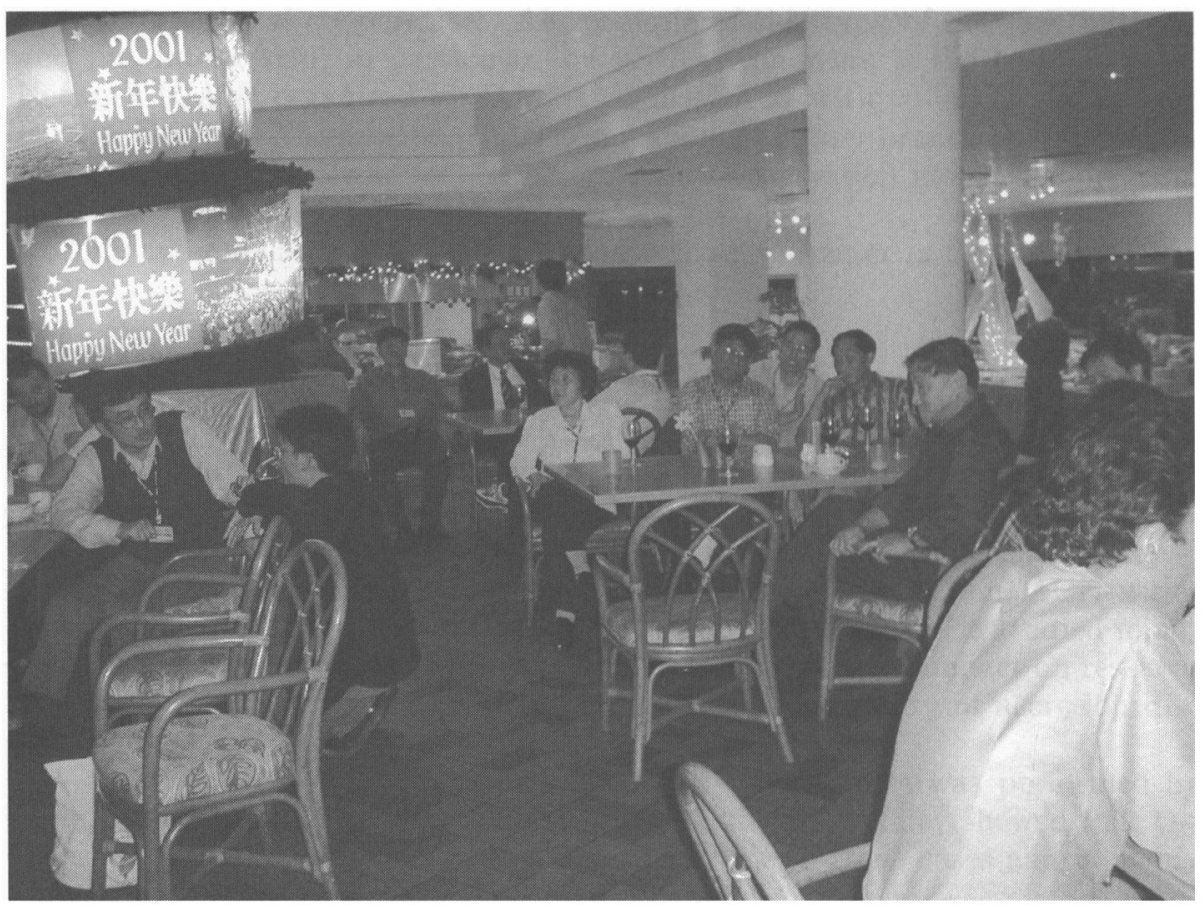

